\title{
Guideline Concordant Care
}

National Cancer Institute

\section{Source}

National Cancer Institute. Guideline Concordant Care. NCI Thesaurus. Code C132336.

Treatment that is in compliance with established guidelines. 